Chirurg 2014 $\cdot 85: 1012$

DOI 10.1007/s00104-014-2903-y

Online publiziert: 12. November 2014

c) Springer-Verlag Berlin Heidelberg 2014
H. Dralle

Universitätsklinik für Allgemein-, Viszeral- und Gefäßchirurgie, Medizinische Fakultät,

Universitätsklinikum Halle, Halle (Saale)

\title{
Transorale Parathyreoidektomie
}

\section{Originalpublikation}

Karakas E, Steinfeldt T, Gockel A et al (2014)

Transoral parathyroid surgery - a new alter-

native or nonsense? Langenbecks Arch Surg 399:741-745

\section{Hintergrund und Fragestellung}

Minimal-invasive Techniken haben in der endokrinen Chirurgie ebenso wie in anderen Bereichen der Chirurgie einen nicht mehr verzichtbaren Stellenwert erlangt. Für einige Bereiche der endokrinen Chirurgie ist das minimal-invasive Vorgehen bereits seit längerem allgemeiner Standard (z. B. Adrenalektomie bei benignen Tumoren und Metastasen; [1]), für andere aktuell deutlich zunehmend (z. B. Insulinome und hormoninaktive neuroendokrine Pankreastumoren; [2]), für andere aber auch trotz erwiesener technischer Machbarkeit nicht ohne Kontroverse (z. B. Roboter-assistierte transaxilläre Schilddrüsenresektion; [3]). Die Autoren haben mit der vorliegenden Studie zur transoralen Parathyreoidektomie (TOP) ein weiteres, überaus kontroverses Thema aufgegriffen und kommen aufgrund ihrer Ergebnisse zu eindeutigen Schlussfolgerungen.

\section{Patienten und Methoden}

Nach Vorliegen des Ethikvotums wurde von 2010 bis 201275 Patienten mit präoperativ benignem sporadischem primärem Hyperparathyreoidismus und positiver Adenomlokalisation eine TOP alternativ zur minimal-invasiven offenen $\mathrm{Pa}$ rathyreoidektomie (PTX) angeboten. Die TOP erfolgte entweder über einen paralingualen Zugang des rechten posterioren Mundbodens oder eine vertikale Inzision im Bereich des Zungenfrenulums.

\section{Ergebnisse}

Nach eingehender Patienteninformation über die Operationsmethode der TOP entschieden sich 5 Patienten (7\%) für das Verfahren. Diejenigen Patienten, die sich trotz des kosmetischen Vorteils gegen die TOP entschieden, waren unsicher hinsichtlich der Sicherheit der Methode oder es war ihnen unangenehm, transoral operiert zu werden. Bei allen 5 Patienten gelang der transorale Zugang zur Schilddrüsenregion, jedoch trotz präoperativ bildgebender Lokalisation bei 2 Patienten keine Auffindung des Nebenschilddrüsenadenoms, sodass eine konventionell-offene PTX erfolgte, die nach 13 bzw. 15 min zur Adenomentfernung führte. Bei einem der beiden Patienten war zur definitiven Heilung wegen Vorliegens einer Mehrdrüsenerkrankung eine weitere konventionell offene PTX erforderlich. Als Operationsfolgen wurden beobachtet: transiente Rekurrensparese [1], transiente Hypoglossusparese (1), permanente Lingualisparese (1), Schmerzen im Operationsgebiet (visuelle Analogskala >7) mit Zungenschwellung (4) und Hämatom (3).

\section{Diskussion und Fazit}

Die Autoren der sorgfältig durchgeführten Studie kommen aufgrund ihrer Ergebnisse zu der eindeutigen Schlussfolgerung, dass die transorale Parathyreoidektomie trotz technischer Machbarkeit aufgrund der geringen Patientenakzeptanz und hohen Komplikationsrate derzeit nicht empfohlen werden kann. Obwohl solitäre Nebenschilddrüsenadenome für die beschriebene Technik prinzipiell eine gute Indikation darstellen würden, ist das gegenwärtig verfügbare Instrumentarium ungeeignet, um einen schon von vornherein aufwen- digen Zugang zu minimieren. Von noch größerer Bedeutung erscheint allerdings, dass das bildgebend lokalisierte Adenom in 2 von 5 Fällen mithilfe der TOP nicht lokalisiert werden konnte, ein Risiko, das bei einem mit herkömmlicher Technik bislang hocheffektiven Eingriff nicht zu unterschätzen ist. Hinzukommt, dass in Zukunft als Alternative zur Operation nichtoperative Verfahren in Entwicklung sind [4], die an Bedeutung gewinnen könnten.

\section{Korrespondenzadresse}

Prof. Dr. Dr. h.c. H. Dralle

Universitätsklinik für Allgemein-, Viszeralund Gefäßchirurgie, Medizinische Fakultät, Universitätsklinikum Halle, Ernst-Grube-Str. 40, 06097 Halle (Saale) henning.dralle@uk-halle.de

Interessenkonflikt. H. Dralle gibt an, dass kein Interessenkonflikt besteht.

\section{Literatur}

1. Moreno $\mathrm{P}$, Quintana Basarrate A de la, Musholt TJ et al (2013) Adrenalectomy for solid tumor metastases: results of a multicenter european study. Surgery 154:1215-1223

2. Dralle H, Satiroglu I (2013) Laparoskopische Chirurgie neuroendokriner Pankreastumoren. Chirurg 84:433

3. Dralle H (2013) Robot-assisted transaxillary thyroid surgery: as safe as conventional-access thyroid surgery? Eur Thyroid J 2:71-75

4. Kovatcheva R, Vlahov J, Stoinov J et al (2014) USguided high-intensity focused ultrasound as a promising non-invasive method for treatment of primary hyperparathyroidism. Eur Radiol 24:20522058 